\title{
Evaluation of Abnormal Pap Smear Distribution in Patients with Various Types of Rheumatologic Diseases
}

\section{Fariba Binesha, Sogol Alsaeedi $^{b}$, Ali Dehghanc, Samira Alsaeedid, Masoomeh Naghshi ${ }^{\mathrm{e}}$ Sedighe Vaziribozorg ${ }^{f}$, Seyed Hossein Shahcheraghi ${ }^{* *}$}

aDepartment of Pathology, Shahid Sadoughi University of Medical Sciences, Yazd, Iran.

${ }^{\mathrm{b}}$ General Physician, Shahid Sadoughi University of Medical Sciences, Yazd, Iran.

'Department of Rheumathology, Shahid Sadoughi University of Medical Sciences, Yazd, Iran.

${ }^{\mathrm{d}}$ Department of Rheumathology, Tehran University of Medical Sciences, Tehran, Iran.

eDepartment of Gynecology, Shahid Sadoughi University of Medical Sciences, Yazd, Iran.

${ }^{\mathrm{f} D e p a r t m e n t}$ of Otolaryngology-Head and Neck Surgery, Otorhinolaryngology Research Center

Shahid Sadoughi University of Medical Sciences, Yazd, Iran.

gInfectious Diseases Research Center, Shahid Sadoughi Hospital, Shahid Sadoughi University of Medical

Sciences, Yazd, Iran.

shahcheraghih@gmail.com

*Corresponding Author: Seyed Hossein Shahcheraghi, Infectious Diseases Research Center, Shahid Sadoughi Hospital, Shahid Sadoughi University of Medical Sciences, Yazd, Iran.

\section{Abstract}

Introduction: Cervical cancer, after breast cancer, is the second most frequent reason of death worldwide. The high load of this disease in developing nations is caused by the absence of screening convenience.This research aimed to evaluate the prevalence of abnormal Pap smears in patients with rheumatologic diseases.

Materials and Methods: In this cross-sectional study 50 women with various rheumatologic diseases were included. The patient's demographic information, including the age, type of rheumatologic disease, duration of the rheumatologic disease, Pap smear findings and types of treatment were recorded in a pre-set questionnaire. The control group composed of 100 married women who were in agreement with the patients' age and other factors but did not have rheumatologic diseases.

Results: The mean age was 44.44 years in the case group and 39.49 in control group. The results showed that of 100 women in the control group 32 had normal pap smears, 67 showed benign reactive changes and 1 showed ASCUS. Among 50 cases that had rheumatologic diseases, 10 had normal Pap smears and 40 showed benign reactive changes. There was no significant difference between abnormal Pap smear in healthy woman and women with rheumatoid diseases. However, benign Pap smears were significantly higher among patients with rheumatoid arthritis and Ankylosing Spondylitis than the other two groups of patients.

Conclusions: According to our results there was not an increased incidence of cervical abnormalities, but it is recommended that women suffering from rheumatologic diseases should be screened for cervical cancer carefully.

Keywords: Pap smear, Rheumatologic disease, Cervical cancer, Healthy woman, Abnormal Pap smear

\section{INTRODUCTION}

Cervical cancer, after breast cancer, is the second most frequent reason of death worldwide [1]. This cancer has a high effect on general health, and majority of these deaths happen in developing states. The high load of this disease in developing nations is caused by the absence of screening convenience and also suboptimal diet and hygiene [2]. Papanicolaoustained (Pap) smear is coined after the pathologist George Papanicolaou, presented the method in 1949 
Evaluation of Abnormal Pap Smear Distribution in Patients with Various Types of Rheumatologic Diseases

[3]. As its presentation, the Pap smear has assisted in decreasing cervical cancer incidence and fatality amounts by half to two-thirds [4]. Risk of malignancy has increased in many rheumatologic diseases in comparison to the general population. Although proof for this increased risk is not clear, it has been presumed that this nexus may be mutual. For example, chronic inflammation that is present in autoimmune disease or its remedies may initiate cancer transformation; on the contrary, anti cancer immune reactions may cause cross-reactive resulting in autoimmunity. A systematic review showed that odds ratio for the risk of high-grade squamous intraepithelial lesions in patients with SLE against controls was 8.66 [5]. Another study revealed that the risk of cervical cancer was considerably higher in SLE patients than control group [6]. In a research of 576 SLE patients, the risk of cancers (virus-related) had increased and this was associated with human papilloma virus (HPV)-related cancers [7]. Immune system dysfunction, disease activity, recurrent infections, and immunosuppressive therapy may be culpable [8]. Given the difficulty in determining infrequent consequences such as malignant tumor in the rheumatologic diseases, large scale epidemiological researches are noteworthy for comprehending the link between these two matters. In this study, we want to understand if patients with rheumalogic diseases are at increased risk of abnormal Pap smears and we also want to highlight the effect of different medications on Pap smear.

\section{Materials ANd Methods}

After approval by local ethics committee and obtaining written informed consent, in this cross-sectional study 50 women with various rheumatologic diseases were included. The files of all married women of childbearing age who have been diagnosed with rheumatic had been extracted and the entire patient's demographic information, including the age, type of rheumatologic disease, duration of the rheumatologic disease, Pap smear findings and types of treatment were recorded in a pre-set questionnaire. The control group was composed of 100 married women who were in agreement with the patients' age and other factors but did not have rheumatologic diseases. In this study, the association between the presence of abnormal Pap smear and rheumatologic diseases was investigated in two groups. Unfortunately, our study had some limitations. The most important of these were the unavailability of information about HPV status and the use of conventional Pap smear instead of liquid base, which increased false negative likelihood. The other one included low sample size.

\section{Statistical Analysis}

Statistical analysis included Chi-Squared test and Fischer's exact test. A p-value $<0.05$ was considered statistically significant.

\section{RESULTS}

The results showed that among the 50 patients with rheumatologic diseases the minimum age was 22 years, the maximum age was 68 years and the mean age was $44.44 \pm 10.98$. In these patients, the shortest duration of the disease was 1 year, with a maximum duration of 30 years and an average of 4.18 \pm 4.87 . The results indicated that of 50 women with rheumatic diseases, 40 (80\%) showed benign reactive changes and 10 (20\%) had normal Pap smear. The statistical test showed that there was no significant difference between abnormal Pap smear in women with rheumatic diseases according to age. However, the highest percentage of people who referred was between the age of 41 and 50 years. In the control population, the minimum age was 18 years, the maximum age was 78 years, the mean was 39.49 \pm 13.49 . The results showed that among 100 cases in the control group 32 had normal pap smears, 67 showed benign reactive changes and 1 showed ASCUS (Table.1).

Table 1. Comparison of Pap smear results between the control and case groups.

\begin{tabular}{|l|l|l|l|l|}
\hline \multirow{2}{*}{ Groups } & \multicolumn{3}{|c|}{ Type of lesion } & \multirow{2}{*}{ Total } \\
\cline { 2 - 4 } & Normal & Benign & Malignant & \\
\hline Case & $(\% 20) 10$ & $(80 \%) 40$ & $(0 \%) 0$ & $50(3 / 33 \%)$ \\
\hline Control & $(32 \%) 32$ & $(67 \%) 67$ & $(1 \%) 1$ & $100(7 / 66 \%)$ \\
\hline Total & $(28 \%) 42$ & $(\% 71.33) 107$ & $\%(006) 1$ & $150(100 \%)$ \\
\hline
\end{tabular}


Evaluation of Abnormal Pap Smear Distribution in Patients with Various Types of Rheumatologic Diseases

The statistical tests showed that there was no significant difference between abnormal Pap smear in the control group according to age. However, the percentage of abnormal Pap smear was increasing

with age. The statistical test showed that there was no significant difference between abnormal Pap smear in women with rheumatic diseases and control group (p-value=0.223) (Table.2).

Table 2. Pap smear results by type of rheumatic disease

\begin{tabular}{|l|l|l|l|l|}
\hline \multirow{2}{*}{ Types of rheumatic diseases } & \multicolumn{2}{|c|}{ Type of lesion } & Total \\
\cline { 3 - 5 } \multicolumn{2}{l|}{} & Normal & Benign & \\
\hline \multirow{3}{*}{ RA } & count & 7 & 29 & 36 \\
\cline { 2 - 5 } & percent & $19.4 \%$ & $80.6 \%$ & $100.0 \%$ \\
\hline \multirow{3}{*}{ SLE } & count & 0 & 3 & 3 \\
\hline \multirow{2}{*}{ AS } & percent & $0.0 \%$ & $100.0 \%$ & $100.0 \%$ \\
\hline \multirow{2}{*}{ Scleroderma } & count & 1 & 7 & 8 \\
\hline \multirow{2}{*}{ Total } & percent & $12.5 \%$ & $87.5 \%$ & $100.0 \%$ \\
\cline { 2 - 5 } & pount & 2 & 1 & 3 \\
\hline
\end{tabular}

The results showed that there was no significant difference between individuals with different types of rheumatologic diseases and their Pap smear type ( $p$-value=0.163); however, benign Pap smears were significantly higher in patients with rheumatoid arthritis and Ankylosing Spondylitis than the other two groups of patients (SLE and Scleroderma). Among these patients 5 patients had normal Pap smears and 9 had benign Pap smears. The duration of the disease in 14 patients was 1 year. The statistical

tests showed that there was no significant difference between abnormal Pap smear and the duration of the disease in patients. Also there were no significant difference between the useage of drugs ( including Prednisolone (p-value $=0.740$ ), Cyclophosphamide (p-value=0.637), Methotrexate (p-value $=0.485)$, Indomethacin ( $\mathrm{p}$-value=0.571), Hydroxychloroquine ( $\mathrm{p}$-value $=0.515), \quad$ Sulfasalazine $\quad(\mathrm{p}$-value $=0.589)$, Azathioprine ( $\mathrm{p}$-value=0.098)) and Pap smear results in women with rheumatologic diseases (Table.3).

Table 3. The relationship between the use of drugs and the result of pap smear in women with rheumatologic diseases. (Prednisolone, Cyclophosphamide, Methotrexate, Indomethacin, Hydroxychloroquine, Sulfasalazine, Azathioprine)

\begin{tabular}{|c|c|c|c|c|c|c|c|c|c|c|c|c|c|}
\hline \multirow{3}{*}{$\begin{array}{l}\text { Type of } \\
\text { lesions }\end{array}$} & \multicolumn{13}{|c|}{ Drugs } \\
\hline & \multicolumn{2}{|c|}{$\mathrm{P}$} & \multicolumn{2}{|c|}{ C } & \multicolumn{2}{|c|}{ M } & \multicolumn{2}{|c|}{ I } & \multicolumn{2}{|c|}{$\mathrm{H}$} & $S$ & \multicolumn{2}{|c|}{ A } \\
\hline & Yes & No & Yes & No & Yes & No & Yes & No & Yes & No & Total & Yes & No \\
\hline Normal & 9 & 1 & 0 & 10 & 8 & 2 & 1 & 9 & 7 & 3 & 4 & 2 & 8 \\
\hline Benign & 36 & 4 & 2 & 38 & 29 & 11 & 6 & 34 & 30 & 10 & 17 & 1 & 39 \\
\hline Total & 45 & 5 & 2 & 48 & 37 & 13 & 7 & 43 & 37 & 13 & 21 & 3 & 47 \\
\hline
\end{tabular}

\section{DISCUSSION}

Cervical cancer has been estimated as the fourth most common cancer in women. Its incidence and mortality is higher in underdeveloped countries. This is a result of more effective screening programs in developed nations [9]. The possibility of abnormal Pap smear with autoimmune disease has been the subject of research in a number of studies, but the results of these studies are not the same. An increased chance of cervical premalignant changes has been found in women with SLE [1]. It is said that this rate is considerably higher amongst women in the first five years of diagnosis [10]. In this study, no association was found between rheumatologic diseases and abnormal Pap smear. 
Evaluation of Abnormal Pap Smear Distribution in Patients with Various Types of Rheumatologic Diseases

One study [1] revealed that four out of 32 SLE patients had abnormal Pap smears in comparison to none cervical abnormalities in the control group. This result is consistent with Esmaeili and Ghahremanzadeh who revealed that the frequency of abnormal Pap smear results was higher in patients with SLE compared to control group [11]. Nath et al. showed that English women with SLE had significantly higher levels of HPV infections and abnormal cervical smears [10]. In a population-based cohort of 441 women with systemic sclerosis [12] 5 cervical cancers were diagnosed among 90 cases of malignant tumor registered. It is in consistent with a Danish nationwide populationbased cohort study conducted by Olesen et al [13]. On the contrary no cases of malignant cervical lesion were found in 2 large cohort studies that were done in Japan [14] and Taiwan [15]. Bernatsky et al [16] assayed the history of an abnormal pap test among 320 females with systemic sclerosis. The prevalence of positive pap smear was higher than healthy women (25.4\% versus $13.8 \%$ ). M. Colaci et al. reported a high frequency of malignant lesions of cervix and vulva in women with systemic sclerosis [17]. A systematic review of 33 studies was done by Santana et al. They deduced that SLE elevates the risk of developing cervical premalignant lesions of uterine cervix. Although the bulk of the researches revealed that females with SLE had more precancerous lesions than control women, the prevalence of cervical cancer was similar between SLE patients and general populations in nearly all studies [18]. Only one survey contained in the review reported an increased prevalence of cervical cancer in SLE patients $[6,18]$. The increased risk of precancerous lesions in this study was certified in a meta-analysis done by Zard et al. [5] This study contained seven studies concerning the prevalence of cervical pre-cancerous in patients with SLE. They reported a 9-fold increment risk of cervical precancerous lesions in these patients [5]. Raposo A et al performed a systematic review analysis of articles about patients with different rheumatologic diseases to investigate the frequency of HPV infection and cervical premalignant and malignant lesions. Among 476 articles found, 27 were included. The studies revealed an augmented prevalence of cervical premalignant and malignant lesions and HPV infection had an important role especially in SLE patients [19].
Some studies reported the effect of diverse treatment used in patients with SLE on the Pap smear results. For example Bateman et al [20] found that there was a considerable decline in time to dysplasia development in patients whom received intravenous cyclophosphamide in comparison to patients without. However, in the current study there was no significant difference between the use of drugs and Pap smear results. The possible reason for the discrepancy between the various studies and the present study may be the inability to understand the main cause of cervical cancer and in particular to isolate the effect of HPV infection. The link between HPV and malignant changes in cervix is now generally accepted. The principle known factor of cervical malignancy is specific genotypes of human papilloma virus infection [17]. By reviewing the articles we can see that in many studies, the role of HPV as an etiologic factor in the pathogenesis of cervical cancer has been proven, but the general belief is that a significant percentage of HPV-infected women will not show malignant changes. HPV infection alone is not adequate to develop cervical premalignant and malignant lesions. The mixture of the specific genotypes of human papilloma virus, its persistence and the reaction of the patient's immune system assign the outcome of the infection [21]. In majority of patients, the immune system is competent to eliminate the virus during several years. As a result only a small percent of infected cases develop clinical complications related to viral infection. Females who are young at the first sexual contact and who have multiple sexual partners have a higher risk to develop HPV related lesions. Smoking, oral contraceptive pills and presence of other sexual transmitted diseases are also important. As well as, immunosuppression or immunodeficiency can assist viral permanence [21]. Another noteworthy point is that the pathogenesis of cervical cancer in Muslim countries differs from that of other Western societies. And this is due to the difference in risk factors. The prevalence of abnormal Pap smear in Iran is much lower than that of western societies. So, an abnormal Pap test in Iran has been reported to be 0.2 to $1 \%$ [22]. This could be related to sexual behavior within the framework of Islamic law. Sexual activity in Iran is only started after marriage, and Iranian religious and cultural customs exclude the likelihood of multiple sexual partners. This in itself 
Evaluation of Abnormal Pap Smear Distribution in Patients with Various Types of Rheumatologic Diseases

reduces the likelihood of HPV infection and other sexually transmitted infections. Other factors, such as circumcision in men and low rate of cigarette smoke among women, can also be effective. Therefore, it is clear from the above that in our society there is a lower risk for HPV infection and consequently less risk for malignant lesions. Therefore, the association between abnormal Pap smear and autoimmune disorders is weaker.

\section{CONCLUSION}

In this study, no association was found between rheumatologic disease and abnormal Pap smear. However Pap smear is a simple, affordable, sound and repeatable method for screening and early detection of cervical malignant lesions in patient with rheumatologic diseases. Given these remarks, although in this study we found no association between rheumatologic disease and abnormal Pap smear; serial cervical screening for premalignant lesions should be performed, since their event might not be so infrequent in the course of rheumatologic diseases.

\section{Acknowledgment}

The authors wish to thank the staff of Infectious Diseases Research Center of Shahid Sadoughi University of Medical Sciences, Yazd.

\section{REFERENCES}

[1] Al-Sherbeni HH, Fahmy AM, Sherif N. Predisposition to cervical atypia in systemic lupus erythematosus: a clinical and cytopathological study. Autoimmune diseases. 2015;2015.

[2] Sarojini N, Srinivasan S, Madhavi Y, Srinivasan S, Shenoi A. The HPV vaccine: science, ethics and regulation. Econ Pol Wkly. 2010;45(27):27-34.

[3] Papanicolaou GN. A survey of the actualities and potentialities of exfoliative cytology in cancer diagnosis. Annals of internal medicine. 1949;31(4):661-74.

[4] Kurman RJ, Henson DE, Herbst AL, Noller $\mathrm{KL}$, Schiffman $\mathrm{MH}$, Bonfiglio $\mathrm{T}$, et al. Interim guidelines for management of abnormal cervical cytology. Jama. 1994;271(23):1866-9.

[5] Zard E, Arnaud L, Mathian A, Chakhtoura Z, Hie $M$, Touraine $P$, et al. Increased risk of high grade cervical squamous intraepithelial lesions in systemic lupus erythematosus: a metaanalysis of the literature. Autoimmunity reviews. 2014;13(7):730-5.

[6] Cibere J, Sibley J, Haga M. Systemic lupus erythematosus and the risk of malignancy. Lupus. 2001;10(6):394-400.

[7] Dreyer L, Faurschou M, Mogensen M, Jacobsen S. High incidence of potentially virus-induced malignancies in systemic lupus erythematosus: A long-term followup study in a Danish cohort. Arthritis \& Rheumatology. 2011;63(10):3032-7.

[8] Ciolkiewicz M, Domyslawska I, Ciolkiewicz A, Klimiuk PA, Kuryliszyn-Moskal A. Coexistence of systemic sclerosis, scleroderma-like syndromes and neoplastic diseases. Polskie Archiwum Medycyny Wewnetrznej. 2008;118(3):119.

[9] Villain P, Gonzalez P, Almonte M, Franceschi S, Dillner J, Anttila A, et al. European code against cancer 4th edition: infections and cancer. Cancer epidemiology. 2015;39:S120-S38.

[10] Nath R, Mant C, Luxton J, Hughes G, Raju KS, ShepherdP,etal.Highriskofhumanpapillomavirus type 16 infections and of development of cervical squamous intraepithelial lesions in systemic lupus erythematosus patients. Arthritis Care \& Research. 2007;57(4):619-25.

[11] Esmaeili H, Ghahremanzadeh K. Association of Pap smear abnormalities with autoimmune disorders. Pakistan Journal of Biological Sciences. 2011;14(10):600.

[12] Hill CL, Nguyen A, Roder D, Roberts-Thomson P. Risk of cancer in patients with scleroderma: a population based cohort study. Annals of the rheumatic diseases. 2003;62(8):728-31.

[13] Olesen AB, Svaerke C, Farkas D, Sørensen HT. Systemic sclerosis and the risk of cancer: a nationwidepopulation-based cohortstudy. British Journal of Dermatology. 2010;163(4):800-6.

[14] Hashimoto A, Arinuma Y, Nagai T, Tanaka S, Matsui $\mathrm{T}$, Tohma $\mathrm{S}$, et al. Incidence and the risk factor of malignancy in Japanese patients with systemic sclerosis. Internal Medicine. 2012;51(13):1683-8.

[15] Kuo C, Luo S, Yu K, Chou I, Tseng W, Chang H, et al. Cancer risk among patients with systemic 
Evaluation of Abnormal Pap Smear Distribution in Patients with Various Types of Rheumatologic Diseases

sclerosis: a nationwide population study in Taiwan. Scandinavian journal of rheumatology. 2012;41(1):44-9.

[16] Bernatsky S, Hudson M, Pope J, Markland J, Robinson D, Jones N, et al. Reports of abnormal cervical cancer screening tests in systemic sclerosis. Rheumatology. 2008;48(2):149-51.

[17] Colaci M, Giuggioli D, Cassone G, Vacchi C, Campomori F, Boselli F, et al. Gynaecological screening for cervical and vulvar malignancies in a cohort of systemic sclerosis patients: our experience and review of the literature. International journal of rheumatology. $2015 ; 2015$.

[18] Santana IU, do Nascimento Gomes A, Lyrio LDC, Grassi MFR, Santiago MB. Systemic lupus erythematosus, human papillomavirus infection, cervical pre-malignant and malignant lesions: a systematic review. Clinical rheumatology. 2011;30(5):665-72.

[19] Raposo A, Tani C, Costa J, Mosca M. Human papillomavirus infection and cervical lesions in rheumatic diseases: a systematic review. Acta reumatologica portuguesa. 2016;41(3):184-90.

[20] Bateman H, Yazici Y, Leff L, Peterson M, Paget S. Increased cervical dysplasia in intravenous cyclophosphamidetreated patients with SLE: a preliminary study. Lupus. 2000;9(7):542-4.

[21] Erickson BK, Alvarez RD, Huh WK. Human papillomavirus: what every provider should know. American journal of obstetrics and gynecology. 2013;208(3):169-75.

[22] Mosavi-jarraahi A, Mohagheghi M, Zeraatti H, Mortazavi H. Cancer registration in Iran asian pacific. Journal of Cancer Prevention, IARC Supplement. 2001;2:35.

Citation: Fariba Binesh, Sogol Alsaeedi, Seyed Hossein Shahcheraghi, et al. Evaluation of Abnormal Pap Smear Distribution in Patients with Various Types of Rheumatologic Diseases. Open Access Journal of Internal Medicine. 2019; 2(1): 1-6.

Copyright: (c) 2019 Fariba Binesh, Sogol Alsaeedi, Seyed Hossein Shahcheraghi, et al. This is an open access article distributed under the Creative Commons Attribution License, which permits unrestricted use, distribution, and reproduction in any medium, provided the original work is properly cited. 